\title{
From analogue to digital for high voltage impulse generator
}

\begin{abstract}
An impulse generator is an electrical apparatus which produces short high-voltage or highcurrent surges. These devices can be classified into two types : (1) impulse voltage generators and (2) impulse current generators. High impulse voltages are used to test the strength of electric power equipment against lightning and switching surges. High impulse currents are needed not only for tests on equipment such as lightning arresters and fuses but also for many other technical applications such as lasers, thermonuclear fusion, and plasma devices. This study focuses on the automation system of the High Voltage (HV) Impulse Generator, currently owned by University Putra Malaysia. The HV impulse generator is currently available in analogue form controlled device whereby it requires a try and error process in order to achieve the high voltage discharge value desired by the users. To ease the users with the process, it is beneficial to make an improvement i.e. transforming the analogue to a digital form of the HV impulse generator. The outcome of this study would enable users to simply key in the desired value and allow the system to do the rest based on the developed program in this study. A controller is developed to enable the system to intelligently adjust the suitable gap distance to discharge the desired high voltage. The programming using $\mathrm{C}$ language has been developed in this study followed by a simulation process. With the use of the programming developed in this study, it was found that the factor which affects the value of the voltage is the gap distance. The test carried out has also proven that having larger gap distance allows higher high voltage discharge value i.e. the relationship is proportional between "gap distance" and "voltage discharge value". In conclusion this study has successfully converted the process from analogue to digital for the High Voltage (HV) Impulse Generator owned by UPM.
\end{abstract}

Keyword: HV impulse generator; Automation system; High voltage discharge; Analogue to digital 